\title{
The Impact of Price Comparison Service on Pricing Strategy in a Dual-Channel Supply Chain
}

\author{
Qi Xu, Zheng Liu, and Bin Shen \\ School of Glorious Sun of Business and Management, Donghua University, 200051 Shanghai, China \\ Correspondence should be addressed to Zheng Liu; liuzheng960@163.com
}

Received 25 July 2013; Revised 12 September 2013; Accepted 23 September 2013

Academic Editor: Pui-Sze Chow

Copyright (C) 2013 Qi Xu et al. This is an open access article distributed under the Creative Commons Attribution License, which permits unrestricted use, distribution, and reproduction in any medium, provided the original work is properly cited.

\begin{abstract}
Recently, price comparison service (PCS) websites are more and more popular due to its features in facilitating transparent price and promoting rational purchase decision. Motivated by the industrial practices, in this study, we examine the pricing strategies of retailers and supplier in a dual-channel supply chain influenced by the signals of PCS. We categorize and discuss three situations according to the signal availability of PCS, under which the optimal pricing strategies are derived. Finally, we conduct a numerical study and find that in fact the retailers and supplier are all more willing to avoid the existence of PCS with the objective of profit maximization. When both of retailers are affected by the PCS, the supplier is more willing to reduce the availability of price information. Important managerial insights are discussed.
\end{abstract}

\section{Introduction}

Price is one of the key competitive dimensions of purchasing a product. As consumers are eager to have access to the better price information in the market, price comparison service (PCS) is naturally born. The PCS provides specific product information and price differences for consumer's reference [1]. By checking the PCS, consumers are able to compare prices with other retailers and make better decisions $[2,3]$. For example, Skyscanner, an online air ticket price comparison website, helps online consumers to compare flight prices of any given route over a month period among different airline and agents. More examples of PCS are summarized in Table 1. According to Table 1, products shown in the PCS include air ticket, hotel, fashion, home product, computer, and electronics. One observation from Table 1 is noteworthy: all products shown in PCS are under fierce price competition.

Price differences and price fluctuations exert an impact on the decision making among consumers and supply chain parties. Huang and Swaminathan [4] compare various products from Amazon and BELK and find that there exists price difference under an online duopoly environment. This observation motivates us to explore the pricing strategy with consideration of PCS. Serenko and Hayes [3] state that the PCS offers tremendous benefits for consumers who may potentially receive lower prices and for online vendors (i.e., retailer), who may not only capture more price information of their rivals but also get more exposure for their brands.

Pricing issues have been extensively studied by the scholars of supply chain; however, the impact of PCS has rarely been investigated. In this study, we first examine how the signals of PCS affect the decision making of supply chain members in a dual-channel supply chain. More specifically, we mainly develop a model with consideration of PCS and assume that supplier's pricing decision is affected by the PCS, while the retailers' pricing decision can be divided into three situations: (i) neither of retailers is affected by the PCS; (ii) either of retailers is affected by the PCS; and (iii) both of retailers are affected by the PCS. According to the three situations mentioned above, we derive the optimal pricing decision of supply chain parties in such a dual-channel supply chain, and our numerical results show that the best strategies for the retailers and supplier are to avoid the existence of PCS. In addition, we find if both of the retailers are affected by the PCS, the supplier should tend to reduce the availability of price information, and if either of retailers is affected by the PCS, the retailer should tend to exclusively cooperate with PCS.

The paper is organized as follows. We show the related literature in Section 2. The model is presented in Section 3, 
TABLE 1: Examples of PCS [3].

\begin{tabular}{|c|c|c|}
\hline Name & URL & Products \\
\hline Become & http://www.become.com/ & $\begin{array}{l}\text { Fashion, electronics, } \\
\text { home, and so forth }\end{array}$ \\
\hline BizRate & http://www.bizrate.com/ & $\begin{array}{l}\text { Fashion, electronics, } \\
\text { home, and so forth }\end{array}$ \\
\hline Boxz & http://www.boxz.com/ & Mobile phones \\
\hline Eprice & http://www.eprice.com.hk/ & Electronics \\
\hline MySimon & http://www.mysimon.com/ & $\begin{array}{l}\text { Fashion and } \\
\text { computer }\end{array}$ \\
\hline PriceGrabber & http://www.pricegrabber.com/ & $\begin{array}{l}\text { Computer and } \\
\text { electronics }\end{array}$ \\
\hline Shopping & http://www.shopping.com/ & $\begin{array}{l}\text { Fashion, electronics, } \\
\text { home, and so forth }\end{array}$ \\
\hline Shopzilla & http://www.shopzilla.com/ & $\begin{array}{l}\text { Fashion, electronics, } \\
\text { home, and so forth }\end{array}$ \\
\hline Smarter & http://www.smarter.com/ & $\begin{array}{l}\text { Fashion, electronics, } \\
\text { home, and so forth }\end{array}$ \\
\hline Skyscanner & http://www.skyscanner.com/ & Air ticket and hotel \\
\hline
\end{tabular}

and we obtain the optimal retail prices on both channels under vertically competition in Section 4. In Section 5, we study the supplier's pricing strategies when facing two competing retailers. We further conduct numerical studies to investigate the impact of PCS on pricing and expected profit among supply chain parties in Section 6. Finally, conclusion is presented in Section 7.

\section{Literature Review}

In the last decade, researchers have studied many issues related to the dual-channel supply chain with traditional and internet channel (please refer to $[5,6]$ for more discussions and review). More recently, Yao and Liu [7] study the pricing competition between retail and e-tail distribution channels under the Bertrand and the Stackelberg price competition models. Interestingly, they find that an optimal wholesale price exists under a different market structure in which the retailer is encouraged to accommodate the additional e-tail channel. Cai [8] examines the impact of channel structure on various supply chain parties with and without coordination under a dual-channel supply chain. Chen et al. [9] investigate the contracting strategies in a dual-channel supply chain. According to their results, the wholesale price contract could coordinate the dual-channel supply chain.

Pricing is popularly investigated in supply chain management, particularly in the dual-channel supply chain [10]. An early research, conducted by Ingene and Parry [11], discusses the case of a single manufacturer selling an identical product to two competing retailers with a linear quantity discount schedule and a two-part tariff. Ingene and Parry claim that coordination is not always in the manufacturer's interest when retailers are competing. Chiang et al. [12] also examine a price competition game in a dual-channel supply chain. They find that a direct channel strategy could lead the manufacturer to be more profitable by posing a viable threat to draw customers away from the retailer. Huang and Swaminathan [4] investigate the pricing strategies in a dual-channel supply chain by assuming a stylized deterministic demand model. Under such demand function, the retailers tend to set a higher retail price in order to make more profit. Recently, Tang and Xing [13] compare the pricing behavior between online branches of traditional retailers and pure internet retailers. They conclude that the price charged by pure e-tailers for DVD titles is $14 \%$ lower than those charged by e-tailers with traditional channels. From the marketing perspective, price comparison can affect buyer's perceptions of acquisition value, transaction value, and behavioral intentions [2]. The impact of PCS on pricing decision is thus inevitable.

The service is significantly important for supply chain management. Dumrongsiri et al. [14] consider the impact of retailer's service quality on a dual-channel supply chain in which a manufacturer sells to a retailer as well as to consumers directly. They reach an interesting conclusion that a higher retailer's service quality may lead to a higher manufacturer's profit in a dual-channel supply chain. Dan et al. [15] examine the retail services in a centralized and a decentralized dual-channel supply chain using the twostage optimization technique and Stackelberg game. Their results imply that retail services could strongly influence the manufacturer and the retailer's pricing strategies. In this paper, we examine the impact of PCS on a dual-channel supply chain. PCS is new to the literature of supply chain management, although it has been largely explored in the area of electronic business $[1,3]$.

The signals of PCS are closely related to information availability, namely, information asymmetry or symmetry. However, information asymmetry has been largely discussed in the literature of supply chain management. Desiraju and Moorthy [16] study information asymmetry regarding a price- and service-sensitive demand curve. They show that the coordination can be achieved by requiring service performance. Cakanyildirim and Sethi [17] find that information asymmetry regarding a manufacturer's production cost does not necessarily cause inefficiency in supply chain. Mukhopadhyay et al. [18] examine the mixed channels under information asymmetry and propose retailer to add differentiated value to the product so as to eliminate the possibility of channel conflict. In this study, we consider both information asymmetry and symmetry cases.

\section{The Model}

In this paper, we consider a dual-channel supply chain system in which one supplier provides the products to two retailers at the same wholesale price, and then two retailers (e.g., WalMart and Amazon) sell products to consumers. Here, we consider one retailer is a physical retailer and the other is an online retailer (As a remark, the physical retailer and the online retailer may or may not be homogenous due to the signal availability of PCS. The heterogeneous structure has particularly happened in the scenario that either of retailers is affected by the PCS). To simplify, the two retailers are referred as retailer1 and retailer2. We consider the retail 


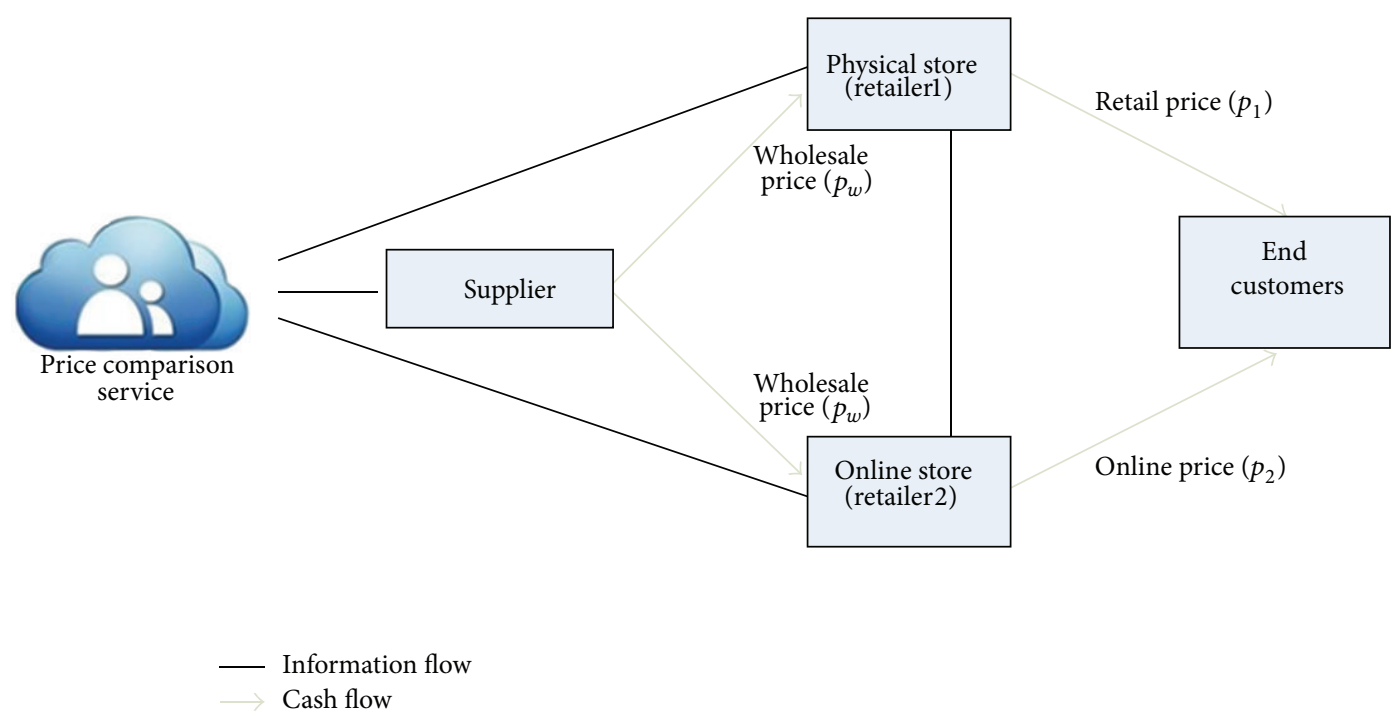

FIGURE 1: Dual-channel supply chain system with PCS.

prices are public information for every party in this supply chain system. All parties face the risk of price competition brought by price comparison service (PCS) (The PCS is a third party who is independent with all parties in such a supply chain), which positively affect their pricing decisions. The sequence of events is as follows. First, supplier decides wholesale price. Second, based on the wholesale price offered by supplier, both retailer1 and retailer2 determine the retail price simultaneously. As a remark, all decisions made by supply chain parties might be influenced by the PCS. This game is analyzed by using backward induction technique. This dual-channel supply chain with the PCS is depicted in Figure 1.

\subsection{Notations}

$D_{1}, D_{2}$ : Market demand for retailer1 and retailer2

$D_{s}$ : Market demand

$p_{w}$ : Wholesale price offered by supplier

$p_{1}, p_{2}$ : Retail prices decided by retailerl and retailer 2 $d$ : Market size base

$\alpha$ : Demand sensitivity for retailerl's market price

$\beta$ : Demand sensitivity for retailer2's market price

$\mu$ : Impact of PCS on demand, which follows a normal distribution $\mu \sim N(0, \sigma)$

$x_{0}, x_{1}, x_{2}$ : Impact of PCS on pricing decision determined by supplier, retailer1, and retailer 2 , respectively

$\theta_{m}, \delta_{m}$ : Adjusted coefficients of retail pricing, $m \in$ $1,2,3,4$

$\lambda_{0}, \lambda$ : Adjusted coefficients of wholesale pricing.

3.2. Basic Model. Without any loss of generality, we consider that the demand curve in the dual-channel supply chain is a linear function of the selling price, where $\mu \sim N\left(0, \sigma^{2}\right)$ is a random parameter to represent random uncertain information [19]. The function of market demand is expressed as follows:

$$
D_{i}\left(p_{i}, p_{j}, \mu\right)=d_{i}-\alpha p_{i}+\beta p_{j}+\mu
$$

where $i, j \in(1,2), i \neq j$, and $d, \alpha, \beta$ are constants.

Supply chain parties might enable to receive the signal of PCS. We denote $x_{0}, x_{1}$, and $x_{2}$ as the degree of PCS signal the supplier and two retailers received, respectively. According to the degree of signal, the parties would make price adjustment, and the adjusted price will in turn affect the market demand. Assuming $x_{i}=\mu+\varepsilon_{i}$, both $\mu$ and $\varepsilon_{i}$ are independent random variables, where $\varepsilon_{i}$ follows normal distribution with mean of $\eta,(\eta>0)$ and variance of $\varepsilon_{i}, i \in 0,1,2$. Covariance matrix of $\varepsilon_{i}$ can be denoted as $\sum=\operatorname{diag}\left(\sigma, s_{0}, s_{1}, s_{2}\right)$. For example, if the price comparison exerts greater impact on retailerl, then the retailerl will adjust its price, which will cause decrease in demand; while if the impact is much less, then the retailerl will tend to keep the price stable, which will not affect the market demand. Prices offered by the supplier and retailers are functions of $x_{0}, x_{1}, x_{2}$, namely, $p_{w}=f_{0}\left(x_{0}\right)$ and $p_{i}=$ $f_{i}\left(x_{i}\right)$.

Supplier adjusts its wholesale price based on the signal of PCS. However, due to asymmetry of transmission, the retailers may or may not be affected by the PCS. Here, we divide it into three situations accordingly.

Situation 1. The supplier does not share price comparison information $x_{0}$ with both of the retailers, namely, neither of retailers are not affected by the PCS information $x_{0}$ directly. Instead, they are able to adjust retail price based on respective price comparison $x_{i}$ as well as wholesale price $p_{w}$. The adjusted retail price hence is $p_{i}=\theta_{1}+\theta_{2} x_{i}+\theta_{3} p_{w}, i \in 1,2$.

Situation 2. PCS information $x_{0}$ exerts impact on either of retailers, then the one who is affected by $x_{0}$ will adjust its price 
and the corresponding retail price is $p_{i}=\theta_{1}+\theta_{2} x_{i}+\theta_{3} p_{w}+$ $\theta_{4} x_{0}, i=1$ or $i=2$.

Situation 3. PCS information $x_{0}$ exerts impact on both of retailers, namely, retailers make pricing decisions based on $x_{0}$ and $x_{i}$. The adjusted retail price is $p_{i}=\theta_{1}+\theta_{2} x_{i}+\theta_{3} p_{w}+\theta_{4} x_{0}$, $i \in 1,2$.

\section{Retail Pricing Strategy}

4.1. Neither of Retailers Is Directly Affected by $x_{0}$. We consider the supplier is affected by price comparison $x_{0}$, and its updated wholesale pricing strategy is

$$
p_{w}=\lambda_{0}+\lambda x_{0},
$$

where $\lambda_{0}, \lambda$ are price coefficients (see [20]). In this subsection, we consider that the supplier does not share information with the two retailers who are only affected by $x_{1}$ and $x_{2}$, respectively. The updated pricing strategies of the two retailers are hence as follows:

$$
\begin{aligned}
& p_{1}=f_{1}\left(x_{1}, p_{w}\right)=\theta_{1}+\theta_{2} x_{1}+\theta_{3} p_{w}, \\
& p_{2}=f_{2}\left(x_{2}, p_{w}\right)=\delta_{1}+\delta_{2} x_{2}+\delta_{3} p_{w} .
\end{aligned}
$$

Assume that the competitive relationship between the two retailers who are well-matched in strength and the impact of PCS on each retailer are equal. Then, the expected demand of retailerl can be expressed as:

$$
E\left(\mu \mid x_{1}, x_{0}\right)=\frac{\sigma s_{0} x_{1}+\sigma s_{1} x_{0}}{\sigma s_{1}+\sigma s_{0}+s_{1} s_{0}} .
$$

According to the above assumption, the formula can be further described as:

$$
\begin{aligned}
E\left(\mu \mid x_{1}, p_{w}\right) & =E\left(x_{2} \mid x_{1}, p_{w}\right) \\
& =\frac{\sigma s_{0} x_{1} \lambda+\sigma s_{1}\left(p_{w}-\lambda_{0}\right)}{\left(\sigma s_{1}+\sigma s_{0}+s_{1} s_{0}\right) \lambda},
\end{aligned}
$$

and the expected demand of retailer 2 can be expressed as:

$$
\begin{aligned}
E\left(\mu \mid x_{2}, p_{w}\right) & =E\left(x_{1} \mid x_{2}, p_{w}\right) \\
& =\frac{\sigma s_{0} x_{2} \lambda+\sigma s_{2}\left(p_{w}-\lambda_{0}\right)}{\left(\sigma s_{2}+\sigma s_{0}+s_{2} s_{0}\right) \lambda} .
\end{aligned}
$$

In addition, the signal of PCS is symmetric for both retailers; the expected prices of retailerl and retailer 2 are

$$
\begin{aligned}
& E\left(p_{2} \mid x_{1}, p_{w}\right)=\delta_{1}+\delta_{2} E\left(x_{2} \mid x_{1}, p_{w}\right)+\delta_{3} p_{w} \\
& E\left(p_{1} \mid x_{2}, p_{w}\right)=\theta_{1}+\theta_{2} E\left(x_{1} \mid x_{2}, p_{w}\right)+\theta_{3} p_{w} .
\end{aligned}
$$

Then, the expected profits of retailer1 and retailer 2 are expressed as:

$$
\begin{array}{r}
E\left(\Pi_{1} \mid x_{1}, p_{w}\right)=\left[d_{1}-\alpha p_{1}+\beta E\left(p_{2} \mid x_{1}, p_{w}\right)\right. \\
\left.+E\left(\mu \mid x_{1}, p_{w}\right)\right]\left(p_{1}-p_{w}\right), \\
E\left(\Pi_{2} \mid x_{2}, p_{w}\right)=\left[d_{2}-\alpha p_{2}+\beta E\left(p_{1} \mid x_{2}, p_{w}\right)\right. \\
\left.+E\left(\mu \mid x_{2}, p_{w}\right)\right]\left(p_{2}-p_{w}\right) .
\end{array}
$$

Proposition 1. When neither of retailers is influenced by the supplier price comparison impact $x_{0}$, the profit functions $E\left(\Pi_{1} \mid x_{1}, p_{w}\right)$ and $E\left(\Pi_{2} \mid x_{2}, p_{w}\right)$ are strictly concave in $p_{1}$ and $p_{2}$, respectively, namely, the optimal retail prices $p_{1}^{*}$ and $p_{2}^{*}$ uniquely exist.

To enhance the presentation, all proofs are relegated to the Appendices.

By solving the first-order derivative of $p_{1}$ in $E\left(\Pi_{1}\right.$ $\left.x_{1}, p_{w}\right)$ and $p_{2}$ in $E\left(\Pi_{1} \mid x_{1}, p_{w}\right)$, respectively, one can have optimal retail pricing strategies of retailerl and retailer 2 in this situation as follows:

$$
\begin{aligned}
& p_{1}^{*}=\frac{1}{2 \alpha}\left[d_{1}+\alpha p_{w}+\beta E\left(p_{2} \mid x_{1}, p_{w}\right)+E\left(\mu \mid x_{1}, p_{w}\right)\right] \\
& p_{2}^{*}=\frac{1}{2 \alpha}\left[d_{2}+\alpha p_{w}+\beta E\left(p_{1} \mid x_{2}, p_{w}\right)+E\left(\mu \mid x_{2}, p_{w}\right)\right] .
\end{aligned}
$$

By solving (2)-(12) simultaneously, one finds $\theta_{i}, i=1,2,3,4$ and $\delta_{i}, i=1,2,3$. Consider

$$
\begin{aligned}
& \theta_{1}=\frac{1-t}{2-t} \\
& -\left(\left(2 \sigma ( 1 - t ^ { 2 } ) \left[4\left(\left(\frac{1}{\alpha+\beta}-d\right) s_{1} k_{2}+2 d s_{2} k_{1}\right)\right.\right.\right. \\
& \left.\left.+d \sigma s_{0}\left(2 s_{1}+t s_{2}\right)\right]\right) \\
& \left.\times\left(V\left(4-t^{2}\right)\right)^{-1}\right), \\
& \theta_{2}=\frac{\sigma s_{0}\left(1-t^{2}\right)\left[(2 /(\alpha+\beta)-2 d) k_{2}+d \sigma s_{0}\right]}{V}, \\
& \theta_{3}=\frac{1}{2-t} \\
& -\left(\left(2 \sigma ( 1 - t ^ { 2 } ) \left[4\left(\left(\frac{1}{\alpha+\beta}-d\right) s_{1} k_{2}+2 d s_{2} k_{1}\right)\right.\right.\right. \\
& \left.\left.+d \sigma s_{0}\left(2 s_{1}+t s_{2}\right)\right]\right) \\
& \left.\times\left(V\left(4-t^{2}\right)\right)^{-1}\right), \\
& \delta_{1}=\frac{1-t}{2-t} \\
& -\left(\left(2 \sigma ( 1 - t ^ { 2 } ) \left[4\left(\left(\frac{1}{\alpha+\beta}-d\right) s_{2} k_{1}+2 d s_{1} k_{2}\right)\right.\right.\right. \\
& \left.\left.+d \sigma s_{0}\left(2 s_{2}+t s_{1}\right)\right]\right) \\
& \left.\times\left(V\left(4-t^{2}\right)\right)^{-1}\right)
\end{aligned}
$$




$$
\begin{gathered}
\delta_{2}=\frac{\sigma s_{0}\left(1-t^{2}\right)\left[(2 /(\alpha+\beta)-2 d) k_{1}+d \sigma s_{0}\right]}{V} \\
\delta_{3}=\frac{1}{2-t} \\
\quad-\left(\left(2 \sigma ( 1 - t ^ { 2 } ) \left[4\left(\left(\frac{1}{\alpha+\beta}-d\right) s_{1} k_{1}+2 d s_{2} k_{2}\right)\right.\right.\right. \\
\left.\left.\quad+d \sigma s_{0}\left(2 s_{2}+t s_{1}\right)\right]\right) \\
\left.\quad \times\left(V\left(4-t^{2}\right)\right)^{-1}\right)
\end{gathered}
$$

where $t=\beta / \alpha, d_{1}=d_{2}=d / 2, k_{1}=\left(\sigma+s_{0}\right) s_{1}+\sigma s_{0}, k_{2}=$ $\left(\sigma+s_{0}\right) s_{2}+\sigma s_{0}$, and $V=4 k_{1} k_{2}-t^{2} \sigma^{2} s_{0}^{2}$.

Thus, the optimal pricing strategies of the two retailers can be obtained by substituting (13) into $p_{1}^{*}=\theta_{1}+\theta_{2} x_{1}+\theta_{3} p_{w}$ and $p_{2}^{*}=\delta_{1}+\delta_{2} x_{2}+\delta_{3} p_{w}$.

4.2. Either of Retailers Is Affected by $x_{0}$. In this situation, either of retailers is affected by price comparison information $x_{0}$. We assume that retailerl is affected by $x_{0}$ while retailer 2 is not affected by $x_{0}$ directly, therefore, we can have

$$
\begin{gathered}
p_{1}=\theta_{1}+\theta_{2} x_{1}+\theta_{3} p_{w}+\theta_{4} x_{0} \\
p_{2}=\delta_{1}+\delta_{2} x_{2}+\delta_{3} p_{w} .
\end{gathered}
$$

Retailerl enables to speculate pricing strategy of retailer2 based on $x_{0}$ and $x_{1}$. Consider

$$
E\left(p_{2} \mid x_{1}, x_{0}\right)=\delta_{1}+\delta_{2} \frac{\sigma s_{0} x_{1}+\sigma s_{1} x_{0}}{\sigma s_{1}+\sigma s_{0}+s_{1} s_{0}}+\delta_{3} p_{w}
$$

But retailer2 enables to speculate pricing strategy of retailer1 based on $x_{2}$ and $p_{w}$. Consider

$$
\begin{gathered}
E\left(p_{1} \mid x_{2}, p_{w}\right)=\theta_{1}+\theta_{2} \frac{\sigma s_{0} x_{2}+\sigma s_{2} p_{w}-\sigma s_{2}}{\sigma s_{2}+\sigma s_{0}+s_{2} s_{0}} \\
+\theta_{3} p_{w}+\theta_{4}\left(p_{w}-1\right) \\
E\left(\mu \mid x_{1}, x_{0}\right)=\frac{\sigma s_{0} x_{1}+\sigma s_{1} x_{0}}{\sigma s_{1}+\sigma s_{0}+s_{1} s_{0}}, \\
E\left(\mu \mid x_{2}, p_{w}\right)=\frac{\sigma s_{0} x_{1} \lambda+\sigma s_{1}\left(p_{w}-\lambda\right)}{\left(\sigma s_{1}+\sigma s_{0}+s_{1} s_{0}\right) \lambda} .
\end{gathered}
$$

In order to reduce potential risks brought by price changes with the signal of PCS, the two retailers determine the optimal retail price by maximizing the expected profit. To this end, the expected profits for retailerl and retailer 2 are

$$
\begin{aligned}
& E\left(\Pi_{1} \mid x_{1}, x_{0}\right) \\
& \quad=E\left[\left(\frac{d}{2}-\alpha p_{1}+\beta p_{2}+\mu\right)\left(p_{1}-p_{w}\right) \mid x_{1}, x_{0}\right], \\
& E\left(\Pi_{2} \mid x_{2}, p_{w}\right) \\
& \quad=E\left[\left(\frac{d}{2}-\alpha p_{2}+\beta p_{1}+\mu\right)\left(p_{2}-p_{w}\right) \mid x_{2}, p_{w}\right] .
\end{aligned}
$$

Proposition 2. When either of retailers is influenced by the supplier price comparison impact $x_{0}$, the profit functions $E\left(\Pi_{1} \mid x_{1}, x_{0}\right)$ and $E\left(\Pi_{2} \mid x_{2}, p_{w}\right)$ are strictly concave in $p_{1}$ and $p_{2}$, respectively, namely, the optimal retail price $p_{1}^{*}$ and $p_{2}^{*}$ uniquely exist.

By solving the first derivative of $p_{1}$ and $p_{2}$, one can have the optimal pricing strategies of the two retailers as follows:

$$
\begin{aligned}
& p_{1}^{*}=\frac{1}{2 \alpha}\left[\frac{d}{2}+\beta E\left(p_{2} \mid x_{1}, x_{0}\right)+E\left(\mu \mid x_{1}, x_{0}\right)+\alpha p_{w}\right] \\
& p_{2}^{*}=\frac{1}{2 \alpha}\left[\frac{d}{2}+\beta E\left(p_{1} \mid x_{2}, p_{w}\right)+E\left(\mu \mid x_{2}, p_{w}\right)+\alpha p_{w}\right]
\end{aligned}
$$

Then, solving (14)-(22) simultaneously, one finds $\theta_{i}, i=$ $1,2,3,4$ and $\delta_{i}, i=1,2,3$. Consider

$$
\begin{aligned}
\theta_{1}= & \frac{1-t}{2-t} \\
& -\frac{\sigma d\left(1-t^{2}\right)\left[2\left(\sigma+s_{0}\right) s_{1} s_{2}+\sigma s_{0}\left(2 s_{2}+t s_{1}\right)\right]}{V(2-t)}
\end{aligned}
$$

$$
\begin{aligned}
\theta_{2}= & \frac{\sigma s_{0}\left(1-t^{2}\right)\left[2(1 /(\alpha+\beta)-d) k_{2}+d \sigma s_{0}\right]}{V}, \\
\theta_{3}= & \frac{1}{2-t} \\
& -\frac{\sigma d\left(1-t^{2}\right)\left[2\left(\sigma+s_{0}\right) s_{1} s_{2}+\sigma s_{0}\left(2 s_{2}+t s_{1}\right)\right]}{\alpha V(2-t)}
\end{aligned}
$$

$$
\theta_{4}=\frac{1}{2-t}-\frac{\alpha \sigma s_{1}\left(1-t^{2}\right)\left(2 k_{2}+t \sigma s_{1}\right)}{V},
$$




$$
\begin{aligned}
& \delta_{1}=\frac{1-t}{2-t} \\
& -\left(\left(4 \sigma^{2}\left(1-t^{2}\right)\left(\frac{1}{\alpha+\beta}-d\right)\right.\right. \\
& \left.\times\left[\left(\frac{2}{\alpha+\beta}-2 d\right)\left(\sigma+s_{0}\right) s_{1} s_{2}+\sigma s_{0}\right]\right) \\
& \left.\times\left(V\left(4-t^{2}\right)\right)^{-1}\right) \text {, } \\
& \delta_{2}=\frac{\sigma s_{0}\left(1-t^{2}\right)\left[(2 /(\alpha+\beta)-2 d) k_{1}+d \sigma s_{0}\right]}{V}, \\
& \delta_{3}=\frac{1}{2-t} \\
& -\left(\left(2 \sigma\left(1-t^{2}\right)\left[2 s_{2}\left(\frac{2}{\alpha+\beta}-2 d\right) k_{1}+d \sigma s_{0}\right]\right.\right. \\
& \left.+d s_{1}\left[\left(\frac{2}{\alpha+\beta}-2 d\right) k_{2}+d \sigma s_{0}\right]\right) \\
& \left.\times\left(\alpha V\left(4-t^{2}\right)\right)^{-1}\right) \text {, }
\end{aligned}
$$

where $t=\beta / \alpha, k_{1}=\left(\sigma+s_{0}\right) s_{1}+\sigma s_{0}, k_{2}=\left(\sigma+s_{0}\right) s_{2}+\sigma s_{0}$, and $V=4 k_{1} k_{2}-t^{2} \sigma^{2} s_{0}^{2}$.

The optimal pricing strategies of the two retailers can be obtained by substituting (22) to (29) into $p_{1}^{*}=\theta_{1}+\theta_{2} x_{1}+$ $\theta_{3} p_{w}+\theta_{4} x_{0}$ and $p_{2}^{*}=\delta_{1}+\delta_{2} x_{2}+\delta_{3} p_{w}$.

4.3. Both of Retailers Are Affected by $x_{0}$. In this situation, the supplier shares price comparison information with retailers, which enables both of retailers to be affected by $x_{0}$ directly. Hence, price functions of retailerl and retailer 2 are as follows:

$$
\begin{aligned}
& p_{1}=\theta_{1}+\theta_{2} x_{1}+\theta_{3} p_{w}+\theta_{4} x_{0}, \\
& p_{2}=\delta_{1}+\delta_{2} x_{2}+\delta_{3} p_{w}+\delta_{4} x_{0} .
\end{aligned}
$$

In this situation, due to price competition, the retailer enables to speculate pricing strategy of its rival according to $x_{1}$ or $x_{2}$. The new pricing strategies of retailerl and retailer2 are

$$
\begin{gathered}
E\left(p_{2} \mid x_{1}, x_{0}\right)=\delta_{1}+\delta_{2} \frac{\sigma s_{0} x_{1}+\sigma s_{1} x_{0}}{\sigma s_{1}+\sigma s_{0}+s_{1} s_{0}} \\
+\delta_{3} p_{w}+\delta_{4} x_{0} \\
E\left(p_{1} \mid x_{2}, x_{0}\right)=\theta_{1}+\theta_{2} \frac{\sigma s_{0} x_{1}+\sigma s_{1} x_{0}}{\sigma s_{1}+\sigma s_{0}+s_{1} s_{0}}+\theta_{3} p_{w}+\theta_{4} x_{0}, \\
E\left(\mu \mid x_{1}, x_{0}\right)=\frac{\sigma s_{0} x_{1}+\sigma s_{1} x_{0}}{\sigma s_{1}+\sigma s_{0}+s_{1} s_{0}} \\
E\left(\mu \mid x_{2}, x_{0}\right)=\frac{\sigma s_{0} x_{2}+\sigma s_{1} x_{0}}{\sigma s_{1}+\sigma s_{0}+s_{1} s_{0}} .
\end{gathered}
$$

The expected profits of retailer 1 and retailer 2 are expressed as:

$$
\begin{aligned}
& E\left(\Pi_{1} \mid x_{1}, x_{0}\right) \\
& \quad=E\left[\left(\frac{d}{2}-\alpha p_{1}+\beta p_{2}+\mu\right)\left(p_{1}-p_{w}\right) \mid x_{1}, x_{0}\right], \\
& E\left(\Pi_{2} \mid x_{2}, x_{0}\right) \\
& \quad=E\left[\left(\frac{d}{2}-\alpha p_{2}+\beta p_{1}+\mu\right)\left(p_{2}-p_{w}\right) \mid x_{2}, x_{0}\right] .
\end{aligned}
$$

Proposition 3. When both of retailers are influenced by the supplier price comparison impact $x_{0}$, the profit functions $E\left(\Pi_{1} \mid x_{1}, x_{0}\right)$ and $E\left(\Pi_{2} \mid x_{2}, x_{0}\right)$ are strictly concave in $p_{1}$ and $p_{2}$, respectively, namely, the optimal retail prices $p_{1}^{*}$ and $p_{2}^{*}$ uniquely exist.

By solving the first derivative of $p_{1}$ and $p_{2}$, one can have the optimal pricing strategies of the two retailers as follows:

$$
\begin{aligned}
& p_{1}^{*}=\frac{1}{2 \alpha}\left[\frac{d}{2}+\beta E\left(p_{2} \mid x_{1}, x_{0}\right)+E\left(\mu \mid x_{1}, x_{0}\right)+\alpha p_{w}\right], \\
& p_{2}^{*}=\frac{1}{2 \alpha}\left[\frac{d}{2}+\beta E\left(p_{1} \mid x_{2}, x_{0}\right)+E\left(\mu \mid x_{2}, x_{0}\right)+\alpha p_{w}\right] .
\end{aligned}
$$

Solving (30)-(38) simultaneously, one finds $\theta_{m}, m=1,2,3,4$ and $\delta_{m}, m=1,2,3,4$. Consider

$$
\begin{gathered}
\theta_{1}=\frac{1-t}{2-t}-\frac{\sigma d\left(1-t^{2}\right)\left[\left(4 \sigma+d s_{0}\right) k_{1}+\sigma s_{0}\left(2 s_{1}+t s_{2}\right)\right]}{U\left(2-t^{2}\right)}, \\
\theta_{2}=\frac{\sigma s_{0}\left(1-t^{2}\right)\left[2(1 /(\alpha+\beta)-d) k_{2}+d \sigma s_{0}\right]}{U}, \\
\theta_{3}=\frac{1}{2-t} \\
\theta_{4}=\frac{\alpha \sigma s\left(1-t^{2}\right)_{0}\left(2 k_{2}+t \sigma s_{0}\right)}{U}, \\
\delta_{1}=\frac{1-t}{2-t} \quad \\
-\left(\left(4 \sigma^{2}\left(1-t^{2}\right)\left(\frac{1}{\alpha+\beta}-d\right)\right.\right. \\
\times\left[\left(\frac{2}{\alpha+\beta}-2 d\right)\left(\sigma+s_{0}\right) s_{1} s_{2}\right. \\
\left.\quad \times\left(U\left(2-t^{2}\right)\right)^{-1}\right) \\
\left.\left.+\sigma s_{0}(2+t)\left(s_{1}+s_{2}\right)\right]\right)
\end{gathered}
$$




$$
\begin{aligned}
& \delta_{2}=\frac{\sigma s_{0}\left(1-t^{2}\right)\left[(2 /(\alpha+\beta)-2 d) k_{1}+d \sigma s_{0}\right]}{U}, \\
& \delta_{3}=\frac{1}{2-t}-\frac{\sigma d\left(1-t^{2}\right)\left[\left(4 \sigma+d s_{0}\right) k_{1}+\sigma s_{0}\left(2 s_{1}+t s_{2}\right)\right]}{V\left(4-t^{2}\right)}, \\
& \delta_{4}=\frac{\alpha \sigma s_{0}\left(1-t^{2}\right)\left(2 k_{1}+t \sigma s_{0}\right)}{U}
\end{aligned}
$$

where $t=\beta / \alpha, k_{1}=\left(\sigma+s_{0}\right) s_{1}+\sigma s_{0}, k_{2}=\left(\sigma+s_{0}\right) s_{2}+\sigma s_{0}$, $V=4 k_{1} k_{2}-t^{2} \sigma^{2} s_{0}^{2}$, and $U=2 k_{1} k_{2}-t^{2} \sigma^{2} s_{0}^{2}$.

The optimal pricing strategies of the two retailers can be obtained by substituting (39) into $p_{1}^{*}=\theta_{1}+\theta_{2} x_{1}+\theta_{3} p_{w}+\theta_{4} x_{0}$ and $p_{2}^{*}=\delta_{1}+\delta_{2} x_{2}+\delta_{3} p_{w}+\delta_{4} x_{0}$.

\section{Wholesale Pricing Strategy}

In this section, we investigate the supplier's pricing decision. The supplier is affected by the signal of PCS. We denote the impact as $x_{0}$. Then, it can be divided into three situations according to previous section, namely, according to whether or not the retailers are affected by $x_{0}$. We consider the wholesale pricing is $p_{w}=\lambda_{0}+\lambda x_{0}$, and the supplier aims to maximize its expected profit

$$
E\left(\Pi_{3} \mid x_{0}\right)=E\left[\left(D_{1}+D_{2}\right) p_{w} \mid x_{0}\right],
$$

where $D_{1}\left(p_{1}, p_{2}, \mu\right)=d_{1}-\alpha p_{1}+\beta p_{2}+\mu, D_{2}\left(p_{1}, p_{2}, \mu\right)=$ $d_{2}-\alpha p_{2}+\beta p_{1}+\mu d_{1}=d_{2}=d / 2$; that is,

$$
\begin{aligned}
& E\left(\Pi_{3} \mid x_{0}\right) \\
& \quad=E\left\{\left[d-(\alpha-\beta)\left(p_{1}+p_{2}\right)+2 \mu\right] p_{w} \mid x_{0}\right\} \\
& \quad=\left\{d-(\alpha-\beta) E\left[\left(p_{1}+p_{2}\right) \mid x_{0}\right]+2 E\left(\mu \mid x_{0}\right)\right\} p_{w}, \\
& E\left(x_{1} \mid x_{0}\right)=E\left(x_{2} \mid x_{0}\right)=E\left(\mu \mid x_{0}\right)=\frac{\sigma}{x_{0}\left(\sigma+s_{0}\right)} .
\end{aligned}
$$

Denoting $\sigma /\left(x_{0}\left(\sigma+s_{0}\right)\right)$ as $\Delta$, optimal wholesale pricing is discussed in the following parts.

5.1. Neither of Retailers Is Affected by $x_{0}$. In this subsection, we consider that $x_{0}$ is not correlated with the two retailers, but retailers are affected indirectly by $x_{0}$ of $p_{w}$. According to (3), (4), and (41), we can get the expected profit of supplier as follows:

$$
\begin{aligned}
& E\left(\Pi_{3} \mid x_{0}\right) \\
& \quad \begin{aligned}
=\left\{d-(\alpha-\beta)\left[\left(\theta_{1}\right.\right.\right. & \left.+\delta_{1}\right)-\left(\theta_{2}+\delta_{2}\right) \Delta \\
& \left.\left.+\left(\theta_{3}+\delta_{3}\right) E\left(p_{w} \mid x_{0}\right)\right]+2 \Delta\right\} p_{w} .
\end{aligned}
\end{aligned}
$$

Proposition 4. When $x_{0}$ exerts impact to neither of retailers, the profit function $E\left(\Pi_{3} \mid x_{0}\right)$ is strictly concave in $p_{w 1}$, and the optimal wholesale price $p_{w 1}^{*}$ uniquely exists.
Solving the first-order derivative of $E\left(\Pi_{3} \mid x_{0}\right)$ with respect to $p_{w}$, one can get

$$
\begin{aligned}
p_{w 1}^{*}= & 8 \alpha d \sigma^{2} s_{0}\left(s_{2}+s_{1}\right)((2-t) \alpha-t \beta+1) \\
& \times(2-t)^{2}\left(1-t^{2}\right)\left(k_{1}+k_{2}\right) \\
& \times\left(\left(\frac{1}{\alpha+\beta}-d\right) s_{1}+2 d s_{2}\left(1+k_{1}\right)\right) \\
& +\frac{\sigma^{2} s_{0}}{\sigma+s_{0}}(2-t)(\alpha-\beta)\left(1-t^{2}\right)\left(k_{1}+k_{2}\right) \\
& \times\left(\left(\frac{2}{\alpha+\beta}-2 d\right)+d \sigma s_{0}\right) \\
& -\frac{(2-t)\left(d\left(\sigma+s_{0}\right)+2 \sigma\right)}{(1-t)\left(\sigma+s_{0}\right)},
\end{aligned}
$$

where $t=\alpha / \beta, \theta_{1}, \delta_{1}, \theta_{2}, \delta_{2}, \theta_{3}, \delta_{3}$ are the same as the results mentioned in Section 4.1.

5.2. Either of Retailers Is Affected by $x_{0}$. In this subsection, we consider either of retailers is affected by $x_{0}$. According to (14), (15), and (41), we can get the expected profit of supplier as follows:

$$
\begin{aligned}
& E\left(\Pi_{3} \mid x_{0}\right) \\
& =\left\{d-(\alpha-\beta)\left[\left(\theta_{1}+\delta_{1}\right)+\left(\theta_{2}+\delta_{2}\right) \Delta+\left(\theta_{3}+\delta_{3}\right)\right.\right. \\
& \left.\left.\quad \times E\left(p_{w} \mid x_{0}\right)+\delta_{4} E\left(x_{0} \mid x_{0}\right)\right]+2 \Delta\right\} p_{w} .
\end{aligned}
$$

Proposition 5. When $x_{0}$ exerts impact on either of the retailers, the supplier's profit function $E\left(\Pi_{3} \mid x_{0}\right)$ is strictly concave in $p_{w 2}$, and the optimal wholesale price $p_{w 2}^{*}$ uniquely exists.

Solving the first-order derivative of $E\left(\Pi_{3} \mid x_{0}\right)$ with respect to $p_{w}$, and let $d E p_{w} / d p_{w}=0$, one can get $p_{w 2}^{*}$

$$
\begin{aligned}
p_{w 2}^{*}=[ & \left(4 k_{1} k_{2}-t^{2} \sigma^{2} s_{0}^{2}\right) \\
& -\left(\left(d\left(4 k_{1} k_{2}-t^{2} \sigma^{2} s_{0}^{2}\right)\left(4-t^{2}\right)\right)\right. \\
& \times\left((1-t)\left(4 k_{1} k_{2}-t^{2} \sigma^{2} s_{0}^{2}\right)-4 \sigma(2-t)\right. \\
& \left.\left.\left.\times\left(2\left(\frac{1}{\alpha+\beta}-d\right) s_{1} k_{2}+d s_{2} k_{1}\right)\right)^{-1}\right)\right] \cdot x_{0} \\
+ & 4 \sigma \alpha((2-t) \alpha-t \beta)\left(1-t^{2}\right)\left(k_{1}+k_{2}\right) \\
\times & \left(\left(2 s_{1}\left(\frac{1}{\alpha+\beta}-d\right)+d s_{2}\right)+d \sigma s_{0}(2+t)\left(s_{1}+s_{2}\right)\right) \\
+ & \frac{2 s_{0} \sigma^{2}}{\sigma+s_{0}}(2-t)(\alpha-\beta)\left(1-t^{2}\right)\left(k_{1}+k_{2}\right)
\end{aligned}
$$




$$
\begin{aligned}
& \times\left(\left(\frac{1}{\alpha+\beta}-d\right)+d \sigma s_{0}\right) \\
& -\left(\left(\sigma s_{1}\left(2 k_{2}+t \sigma s_{1}\right)(1+t)\right)\right. \\
& \times\left(\left(4 k_{1} k_{2}-t^{2} \sigma^{2} s_{0}^{2}\right)\left(4-t^{2}\right)-4 \sigma(2-t)(1+t)\right. \\
& \left.\left.\times\left(2\left(\frac{1}{\alpha+\beta}-d\right) s_{1} k_{2}+d s_{2} k_{1}\right)\right)^{-1}\right),
\end{aligned}
$$

where $t=\alpha / \beta, \theta_{1}, \delta_{1}, \theta_{2}, \delta_{2}, \theta_{3}, \delta_{3}$ are the same as the results in Section 4.2 .

5.3. Both of Retailers Are Affected by $x_{0}$. In this subsection, $x_{0}$ exerts impact on both of retailers. According to (30), (31), and (41), we can get the expected profit of supplier as follows:

$$
\begin{aligned}
E\left(\Pi_{3} \mid x_{0}\right) & \\
=\{d-(\alpha-\beta)[ & \left(\theta_{1}+\delta_{1}\right)+\left(\theta_{2}+\delta_{2}\right) \Delta+\left(\theta_{3}+\delta_{3}\right) \\
& \times E\left(p_{w} \mid x_{0}\right) \\
& \left.\left.+\left(\theta_{4}+\delta_{4}\right) E\left(x_{0} \mid x_{0}\right)\right]+2 \Delta\right\} p_{w} .
\end{aligned}
$$

Proposition 6. When $x_{0}$ exerts impact on both of retailers, the profit function $E\left(\Pi_{3} \mid x_{0}\right)$ is strictly concave in $p_{w 3}$, and the optimal wholesale price $p_{w 3}^{*}$ uniquely exists.

Solving the first-order derivative of $E\left(\Pi_{3} \mid x_{0}\right)$ with respect to $p_{w}$, one obtains the optimal wholesale price $p_{w 3}^{*}$ as follows:

$$
\begin{aligned}
p_{w 3}^{*}= & \frac{\alpha \sigma s_{0}\left(1-t^{2}\right)\left(2\left(k_{1}+k_{2}\right)+t \sigma s_{0}\right)}{2 k_{1} k_{2}-t^{2} \sigma^{2} s_{0}^{2}} \cdot x_{0} \\
+ & +\left(\alpha-\frac{1}{d}\right) \\
& \times\left[\left(4 \alpha \sigma^{2}\left(1-t^{2}\right)\left(\frac{1}{\alpha+\beta}-d\right)\right.\right. \\
& \times\left(\left(\frac{2}{\alpha+\beta}-2 d\right)+(2+t) \sigma s_{0}\left(s_{1}+s_{2}\right)\right) \\
& +\alpha \sigma^{2} d\left(1-t^{2}\right) \\
& \left.\times\left(\left(4 \sigma+d s_{0}\right) k_{1}+\sigma s_{0}\left(2 s_{1}+s_{2}\right)\right)\right)
\end{aligned}
$$

$$
\begin{aligned}
& \times\left(\alpha\left(2 k_{1} k_{2}-t^{2} \sigma^{2} s_{0}^{2}\right)\left(2-t^{2}\right)\right. \\
& \left.\left.\times\left(4 k_{1} k_{2}-t^{2} \sigma^{2} s_{0}^{2}\right)\left(4-t^{2}\right)\right)^{-1}\right)-\frac{\sigma}{\sigma+s_{0}} \\
& \left.. \frac{\left(1-t^{2}\right) \sigma s_{0}\left((2 /(\alpha+\beta)-2 d)\left(k_{1}+k_{2}\right)+d s_{0}\right)}{2 k_{1} k_{2}-t^{2} \sigma^{2} s_{0}^{2}}\right],
\end{aligned}
$$

where $t=\alpha / \beta, \theta_{1}, \delta_{1}, \theta_{2}, \delta_{2}, \theta_{3}, \delta_{3}$ are the same as the results in Section 4.3.

\section{Simulation Analysis}

Assuming that $d=200, \alpha=0.5, \beta=0.3, \operatorname{diag}\left(\sigma, s_{0}, s_{1}, s_{2}\right)=$ $\operatorname{diag}(10,2,4,3)$, and $\lambda=10, \lambda_{0}=40$ based on the three situations discussed above, we make an analysis of how $x_{0}, x_{1}, x_{2}$ affects optimal pricing $p_{1}^{*}, p_{2}^{*}, p_{w}^{*}$. Based on the analysis of the three situations shown above, we are able to obtain optimal wholesale prices in different situations. The numerical results are shown in Tables 2, 3, 4, and 5.

6.1. Simulation Analysis of Retail Pricing Model. Recall that $x_{0}, x_{1}, x_{2}$ represent the degree of PCS signal on price, we take the values of $x_{0}, x_{1}, x_{2}$ from 0 to 1 , where $x_{0}, x_{1}, x_{2}=0$ implies that the corresponding parties are not affected by the signal of PCS, whereas $x_{0}, x_{1}, x_{2}=1$ implies that the corresponding party are fully affected by the signal of PCS. As for the retailers, from Figure 2, we can see when $x_{0}, x_{1}, x_{2}$ take values from 0 to 1 , the line of Situation $1 p_{1}^{*}$ is higher than those in Situations 2 and 3; the line of Situation $2 p_{2}^{*}$ is higher than the line of Situation $2 p_{1}^{*}$; the line of Situation $3 p_{1}^{*}$ is the lowest one among all situations. Besides, optimal prices in every situation are increasing with $x_{0}, x_{1}, x_{2}$. From Figure 3, we can find that the line of Situation $1 E\left(p_{1}^{*}\right)$ is the highest among all situations. When $x_{0}, x_{1}, x_{2}$ are from 0.4 to 0.7 , the line of Situation $2 E\left(p_{1}^{*}\right), E\left(p_{2}^{*}\right)$ and the line of Situation $3 E\left(p_{1}^{*}\right)$ are getting closer. Besides, optimal profits of every situation are increasing with $x_{0}, x_{1}, x_{2}$.

According to Figures 2 and 3, three observations for the retailers are noteworthy.

Observation 1. The optimal retail price of Situation 1 and its corresponding optimal profit are higher than those of Situations 2 and 3. In other words, in order to obtain a higher profit, the retailers are more willing to avoid the existence of PCS.

Observation 2. Although the retailers tend to avoid the existence of PCS, they are also willing to seek more availability of price information because the optimal retail price and their corresponding profit would be higher if the availability of pricing information is more sufficient.

Observation 3. In Situation 2, obtaining the information from the PCS could lead retailerl to set a higher retail price and gain more profits than those of retailer2. Hence, the retailer should 
TABLE 2: Optimal retail pricing strategies in different situations.

\begin{tabular}{lcccccccccccc}
\hline & & \multicolumn{1}{c}{$x_{0}=x_{1}=x_{2}$} & & & & \\
& & 0 & 0.1 & 0.2 & 0.3 & 0.4 & 0.5 & 0.6 & 0.7 & 0.8 & 0.9 & 1 \\
\hline Situation 1 & $p_{1}^{*}, p_{2}^{*}$ & 174 & 176 & 178 & 180 & 183 & 187 & 189 & 193 & 198 & 201 \\
Situation 2 & $p_{1}^{*}$ & 96 & 103 & 111 & 120 & 125 & 130 & 134 & 140 & 147 & 152 & 158 \\
& $p_{2}^{*}$ & 150 & 156 & 160 & 163 & 166 & 169 & 172 & 174 & 176 & 178 & 181 \\
Situation 3 & $p_{1}^{*}, p_{2}^{*}$ & 71 & 76 & 80 & 89 & 101 & 111 & 120 & 126 & 138 & 147 & 152 \\
\hline
\end{tabular}

TABLE 3: Optimal profits in different situations.

\begin{tabular}{lcccccccccccc}
\hline & & \multicolumn{1}{c}{$x_{0}=x_{1}=x_{2}$} & & & & \\
& & 0 & 0.1 & 0.2 & 0.3 & 0.4 & 0.5 & 0.6 & 0.7 & 0.8 & 0.9 & 1 \\
\hline Situation 1 & $E\left(p_{1}^{*}\right), E\left(p_{2}^{*}\right)$ & 22358 & 22248 & 22411 & 22468 & 22712 & 23089 & 23194 & 23564 & 24060 & 24289 & 24645 \\
Situation 2 & $E\left(p_{1}^{*}\right)$ & 7918 & 8083 & 8289 & 8540 & 8649 & 9758 & 9829 & 9985 & 10180 & 10301 & 10453 \\
& $E\left(p_{2}^{*}\right)$ & 6432 & 6908 & 7382 & 7845 & 8071 & 8384 & 8624 & 8909 & 9516 & 9918 & 10099 \\
Situation 3 & $E\left(p_{1}^{*}\right), E\left(p_{2}^{*}\right)$ & 5659 & 5968 & 6192 & 6781 & 7548 & 8134 & 8444 & 8644 & 8850 & 8972 & 9132 \\
\hline
\end{tabular}

TABLE 4: Optimal wholesale price offered by supplier under different $x_{0}$.

\begin{tabular}{cccccccccccc}
\hline \multicolumn{10}{c}{$x_{0}$} \\
& 0 & 0.1 & 0.2 & 0.3 & 0.4 & 0.5 & 0.6 & 0.7 & 0.8 & 0.9 & 1 \\
\hline$p_{w 1}^{*}$ & 150 & 150 & 150 & 150 & 150 & 150 & 150 & 150 & 150 & 150 & 150 \\
$p_{w 2}^{*}$ & 167 & 182 & 197 & 212 & 227 & 242 & 257 & 272 & 287 & 302 & 317 \\
$p_{w 3}^{*}$ & 233 & 273 & 313 & 353 & 393 & 433 & 473 & 513 & 553 & 593 & 633 \\
\hline
\end{tabular}

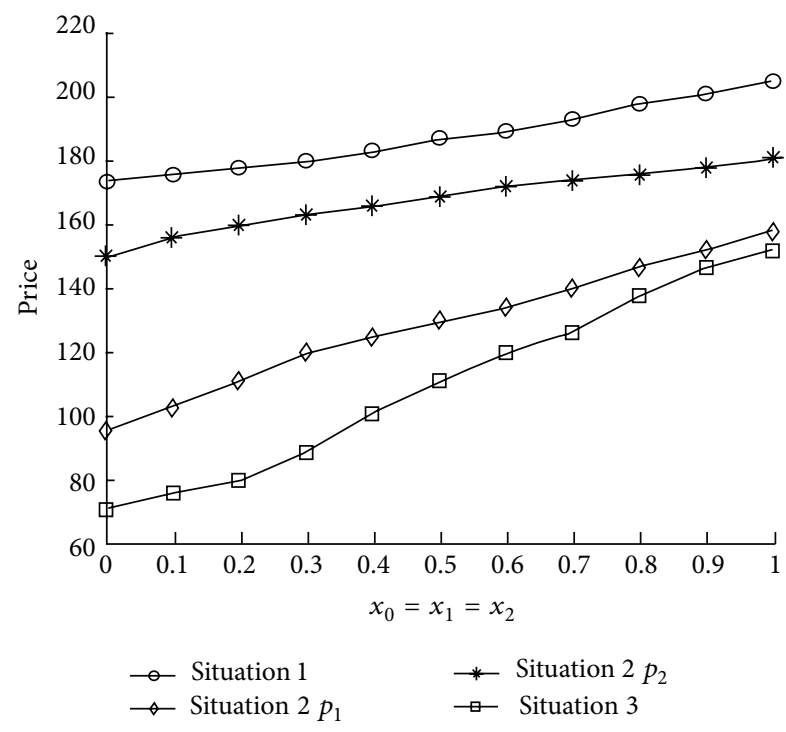

FIgURE 2: Optimal retail prices for 4.1, 4.2, and 4.3.

tend to exclusively cooperate with the PCS. This observation enhances the value of the existence of PCS.

Based on Observations 1, 2, and 3, the best strategy for the retailers is to avoid the PCS and meanwhile to increase the availability of price information. In reality, the PCS might be a third party service who is out of the control of the retailers.

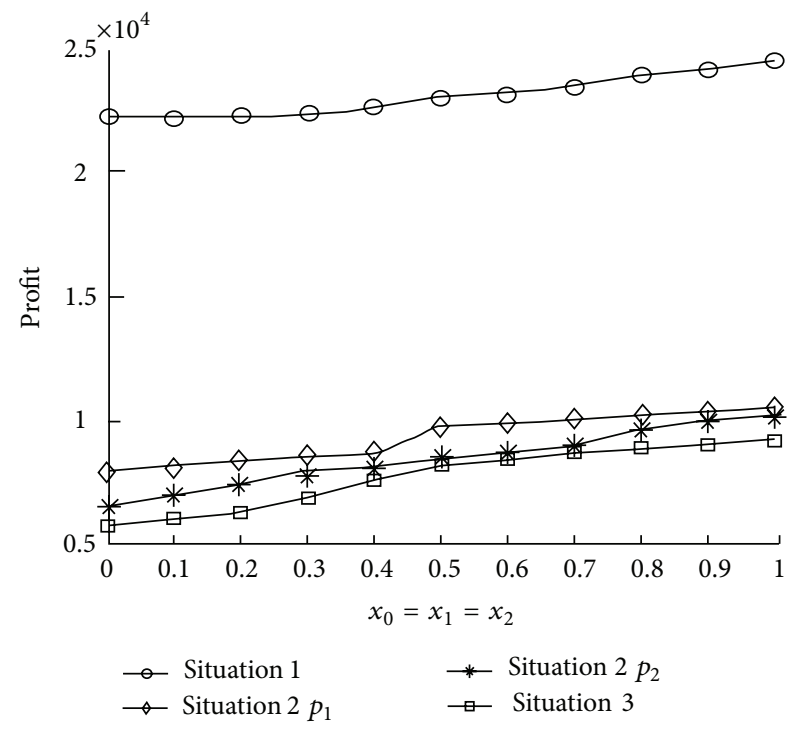

FIGURE 3: Optimal profits of retailers for 4.1, 4.2, and 4.3.

However, our results provide an important implication about how the retailers react if the PCS existed.

6.2. Simulation Analysis of Wholesale Pricing Model. As for the supplier, from Figure 4, we can see that the wholesale prices in every situation are linearly correlated with $x_{0}$. For certain $x_{0}$, the optimal wholesale prices in Situation 3 is higher than those in Situations 1 and 2. And the optimal wholesale prices in Situations 2 and 3 are increasing with $x_{0}$. From Figure 5, we can find that profits in Situations 2 and 3 are increasing with $x_{0}$; however, the one in Situation 1 is linearly negatively correlated with $x_{0}$.

According to Figures 4 and 5, three observations are noteworthy. 
TABLE 5: Optimal profits of supplier under different $x_{0}$.

\begin{tabular}{lccccccccccc}
\hline & & \multicolumn{1}{c}{$x_{0}$} & & & & \\
& 0 & 0.1 & 0.2 & 0.3 & 0.4 & 0.5 & 0.6 & 0.7 & 0.8 & 0.9 & 1 \\
\hline$E\left(p_{w 1}^{*}\right)$ & 23400 & 22050 & 21300 & 20100 & 18450 & 17250 & 16350 & 15150 & 14100 & 13200 & 12150 \\
$E\left(p_{w 2}^{*}\right)$ & 27555 & 27664 & 28171 & 29468 & 29964 & 30976 & 31611 & 32096 & 32431 & 32616 & 33285 \\
$E\left(p_{w 3}^{*}\right)$ & 34018 & 38493 & 41316 & 43713 & 45195 & 46764 & 47773 & 49248 & 51429 & 52777 & 53805 \\
\hline
\end{tabular}

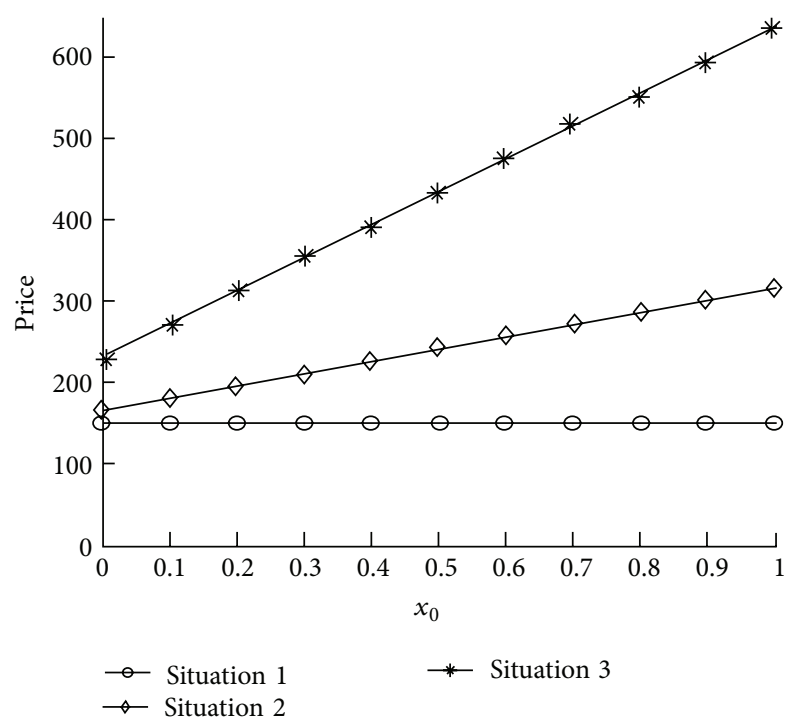

FIGURE 4: Optimal wholesale prices for 5.1, 5.2, and 5.3.

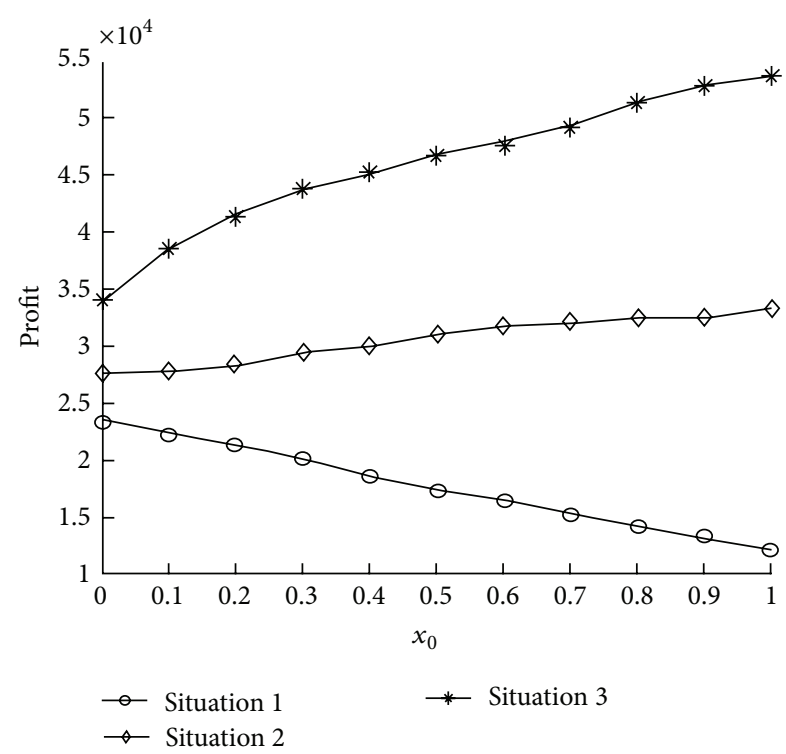

Figure 5: Optimal profits of supplier for 5.1, 5.2, and 5.3.

Observation 4. The optimal wholesale price of Situation 1 is lower than those of Situations 1 and 2, while the corresponding optimal profit is higher than those of Situations 1 and 2. This observation implies that the supplier tends to set a higher wholesale price if the PCS affects the decisions of retail price made by both of retailers. Similar to the results of retailers, the supplier is also more willing to avoid the existence of PCS.

Observation 5. We notice that, in Situation 3, both of the retailers are affected by the PCS; more availability of price information would lead to a decrease in the supplier's profit. Hence, the supplier in Situation 3 should keep the availability of price information as much as possible.

Observations 4 and 5 indicate that the supplier is more willing to avoid the impact of PCS and also increase the availability of price information; however, if both of the retailers are affected by the PCS, he should tend to reduce the availability of price information.

\section{Conclusions}

With the rapid development of e-commerce, the PCS enables to provide transparent price and promote rational consumption. However, the PCS would also affect the pricing decision made by supply chain parties. In this study, we derived the optimal pricing strategies with respective to three situations of signal availability of PCS. Our numerical study implied that when both of retailers are affected by the PCS, the supplier is more willing to reduce the availability of price information, and when either of retailers is affected by the PCS, the retailer should tend to exclusively cooperate with the PCS. In addition, the retailers and supplier are all more willing to avoid the existence of PCS and increase the availability of price information. This result implied that (1) if the entire supply chain belongs to an identical corporate, the corporate is better to merge the PCS; (2) more transparency in price information actually hurts the supply chain parties' and supply chain's profit. This is consistent with the industrial practice in which price war could lead to a big drop in both market and price [21].

For the research limitations, we admit that the findings are mainly based on the analytical models and numerical studies we have developed under various assumptions, such as a linear demand function with respect to the PCS. In addition, the analysis is conducted on a relatively simple dualchannel supply chain of one supplier and two competing retailers, which is an abstract version of the more complex real world supply chain. In this paper, we consider that the PCS is independent from the parties in supply chain; however, according to the practices in China, we observe that Baidu has developed new business model as a reaction to price comparison impact. It would be interesting to investigate the mechanism of how the supply chain parties should cooperate with the PCS. It is also promising to extend 
the model and the analysis to more complex supply chain systems (such as the ones that are risk averse agents [22-24]). It is also meaningful to investigate whether it is beneficial for the supplier to always share information from contracting perspective among supply chain parties $[25,26]$. This would address another interesting topic from the perspective of social influence on the PCS in supply chain management [27].

\section{Appendices}

Proof of Proposition 1. According to (2), (4), (6), (8), and (10), we have

$$
\begin{aligned}
E( & \left.\Pi_{1} \mid x_{1}, p_{w}\right) \\
= & {\left[d_{1}-\alpha p_{1}+\beta E\left(p_{2} \mid x_{1}, p_{w}\right)+E\left(\mu \mid x_{1}, p_{w}\right)\right] } \\
& \times\left(p_{1}-p_{w}\right) \\
= & {\left[d_{1}-\alpha p_{1}+\beta\left(\delta_{1}+\delta_{2} E\left(x_{2} \mid x_{1}, p_{w}\right)+\delta_{3} p_{w}\right)\right] } \\
& \times\left(p_{1}-p_{w}\right) \\
= & {\left[d_{1}-\alpha p_{1}\right.} \\
& \left.+\beta\left(\delta_{1}+\delta_{2} \cdot \frac{\sigma s_{0} x_{2} \lambda+\sigma s_{2}\left(p_{w}-\lambda_{0}\right)}{\left(\sigma s_{2}+\sigma s_{0}+s_{2} s_{0}\right) \lambda}+\delta_{3} p_{w}\right)\right] \\
& \left.\left.\left.\times\left(p_{1}-p_{w}\right)\right]+\delta_{3}\left(\lambda_{0}+\lambda x_{0}\right)\right)\right]\left[p_{1}-\left(\lambda_{0}+\lambda x_{0}\right)\right] \\
= & {\left[d_{1}-\alpha p_{1}+\beta\left(\delta_{1}+\delta_{2} \cdot \frac{\sigma s_{0} x_{2}+\sigma s_{2} x_{0}}{\sigma s_{2}+\sigma s_{0}+s_{2} s_{0}}\right.\right.} \\
= & -\alpha p_{1}^{2}+W_{1} p_{1}+Y_{1},
\end{aligned}
$$

where $\alpha>0, W_{1}=d_{1}+\beta\left(\delta_{1}+\delta_{2} \cdot\left(\sigma s_{0} x_{2}+\sigma s_{2} x_{0}\right) /\left(\sigma s_{2}+\sigma s_{0}+\right.\right.$ $\left.\left.s_{2} s_{0}\right)+\delta_{3}\left(\lambda_{0}+\lambda x_{0}\right)\right)+\alpha\left(\lambda_{0}+\lambda x_{0}\right)$ and $Y_{1}=-\left(\lambda_{0}+\lambda x_{0}\right)\left(d_{1}+\right.$ $\left.\beta\left(\delta_{1}+\delta_{2} \cdot\left(\sigma s_{0} x_{2}+\sigma s_{2} x_{0}\right) /\left(\sigma s_{2}+\sigma s_{0}+s_{2} s_{0}\right)+\delta_{3}\left(\lambda_{0}+\lambda x_{0}\right)\right)\right)$.

According to (2), (3), (7), (9), and (11), we have

$$
\begin{aligned}
E\left(\Pi_{2} \mid x_{2}, p_{w}\right) & {\left[d_{2}-\alpha p_{2}+\beta E\left(p_{1} \mid x_{2}, p_{w}\right)\right.} \\
& \left.+E\left(\mu \mid x_{2}, p_{w}\right)\right]\left(p_{2}-p_{w}\right) \\
= & {\left[d_{2}-\alpha p_{2}+\beta \frac{\lambda \sigma s_{0} x_{2}+\sigma s_{2}\left(p_{w}-\lambda_{0}\right)}{\left(\sigma s_{2}+\sigma s_{0}+s_{2} s_{0}\right) \lambda}\right.} \\
& \left.+\frac{\lambda \sigma s_{0} x_{2}+\sigma s_{2}\left(p_{w}-\lambda_{0}\right)}{\left(\sigma s_{2}+\sigma s_{0}+s_{2} s_{0}\right) \lambda}\right]\left(p_{2}-p_{w}\right)
\end{aligned}
$$

$$
\begin{aligned}
& =\left[d_{2}-\alpha p_{2}+\beta \frac{\sigma s_{0} x_{2}+\sigma s_{2}}{\sigma s_{2}+\sigma s_{0}+s_{2} s_{0}}\right. \\
& \left.\quad+\frac{\sigma s_{0} x_{2}+\sigma s_{2}}{\sigma s_{2}+\sigma s_{0}+s_{2} s_{0}}\right]\left[p_{2}-\left(\lambda_{0}+\lambda x_{0}\right)\right] \\
& =-\alpha p_{2}^{2}+W_{2} p_{2}+Y_{2},
\end{aligned}
$$

where $\alpha>0, W_{2}=\alpha\left(\lambda_{0}+\lambda x_{0}\right)\left(d_{2}+\beta\left(\sigma s_{0} x_{2}+\sigma s_{2}\right) /\left(\sigma s_{2}+\right.\right.$ $\left.\left.\sigma s_{0}+s_{2} s_{0}\right)+\left(\sigma s_{0} x_{2}+\sigma s_{2}\right) /\left(\sigma s_{2}+\sigma s_{0}+s_{2} s_{0}\right)\right), Y_{2}=-\left(\lambda_{0}+\right.$ $\left.\lambda x_{0}\right)\left(\beta\left(\left(\sigma s_{0} x_{2}+\sigma s_{2}\right) /\left(\sigma s_{2}+\sigma s_{0}+s_{2} s_{0}\right)\right)+\left(\sigma s_{0} x_{2}+\sigma s_{2}\right) /\left(\sigma s_{2}+\right.\right.$ $\left.\left.\sigma s_{0}+s_{2} s_{0}\right)\right)$.

According to the nature of the quadratic function and $Y_{2}<0$, the profit functions $E\left(\Pi_{1} \mid x_{1}, p_{w}\right)$ and $E\left(\Pi_{2} \mid x_{2}, p_{w}\right)$ are strictly concave in $p_{1}$ and $p_{2}$, respectively.

Proof of Proposition 2. According to (2), (15), (18), and (20), we have

$$
\begin{aligned}
& E(\left.\Pi_{1} \mid x_{1}, x_{0}\right) \\
&=E {\left[\left(\frac{d}{2}-\alpha p_{1}+\beta p_{2}+\mu\right)\left(p_{1}-p_{w}\right) \mid x_{1}, x_{0}\right] } \\
&=E\left[\left(\frac{d}{2}-\alpha p_{1}+\beta\left(\delta_{1}+\delta_{2} x_{2}+\delta_{3}\left(\lambda_{0}+\lambda x_{0}\right)+\delta_{4} x_{0}\right)\right.\right. \\
&\left.\quad+\mu)\left(p_{1}-\lambda_{0}-\lambda x_{0}\right) \mid x_{1}, x_{0}\right] \\
&=-\alpha p_{1}^{2}+W_{3} p_{1}+Y_{3},
\end{aligned}
$$

where $\alpha>0, W_{3}$ represents a coefficient, and $Y_{3}$ represents constant in this quadratic function.

According to (2), (14), (17), and (19), we have

$$
\begin{aligned}
& E(\left.\Pi_{2} \mid x_{2}, p_{w}\right) \\
&=E {\left[\left(\frac{d}{2}-\alpha p_{2}+\beta p_{1}+\mu\right)\left(p_{2}-p_{w}\right) \mid x_{2}, p_{w}\right] } \\
&=E\left[\left(\frac{d}{2}-\alpha p_{2}+\beta\left(\theta_{1}+\theta_{2} x_{1}+\theta_{3} p_{w}+\theta_{4} x_{0}\right)+\mu\right)\right. \\
&\left.\quad \times\left(p_{2}-\lambda_{0}-\lambda x_{0}\right) \mid x_{2}, p_{w}\right] \\
&=-\alpha p_{2}^{2}+W_{4} p_{2}+Y_{4},
\end{aligned}
$$

where $\alpha>0, W_{4}$ represents a coefficient, and $Y_{4}$ represents constant in this quadratic function.

Similar to Proposition 1, according to the nature of the quadratic function, both $E\left(\Pi_{1} \mid x_{1}, x_{0}\right)$ and $E\left(\Pi_{2} \mid x_{2}, p_{w}\right)$ are strictly concave in $p_{1}$ and $p_{2}$, respectively. 
Proof of Proposition 3. According to (2), (31), (32), (34), and (36), we have

$$
\begin{aligned}
& E(\left.\Pi_{1} \mid x_{1}, x_{0}\right) \\
&=E {\left[\left(\frac{d}{2}-\alpha p_{1}+\beta p_{2}+\mu\right)\left(p_{1}-p_{w}\right) \mid x_{1}, x_{0}\right] } \\
&=E {\left[\left(\frac{d}{2}-\alpha p_{1}+\beta\left(\delta_{1}+\delta_{2} x_{2}+\delta_{3} p_{w}+\delta_{4} x_{0}\right)+\mu\right)\right.} \\
&\left.\quad \times\left(p_{1}-\lambda_{0}-\lambda x_{0}\right) \mid x_{1}, x_{0}\right] \\
&=-\alpha p_{1}^{2}+W_{5} p_{1}+Y_{5},
\end{aligned}
$$

where $\alpha>0, W_{5}$ represents a coefficient, and $Y_{5}$ represents constant in this quadratic function.

According to (2), (30), (33), and (35), we have

$$
\begin{aligned}
E( & \left.\Pi_{2} \mid x_{2}, x_{0}\right) \\
=E & {\left[\left(\frac{d}{2}-\alpha p_{2}+\beta p_{1}+\mu\right)\left(p_{2}-p_{w}\right) \mid x_{2}, x_{0}\right] } \\
=E & {\left[\left(\frac{d}{2}-\alpha p_{2}+\beta\left(\theta_{1}+\theta_{2} x_{1}+\theta_{3} p_{w}+\theta_{4} x_{0}\right)+\mu\right)\right.} \\
& \left.\times\left(p_{2}-\lambda_{0}-\lambda x_{0}\right) \mid x_{2}, x_{0}\right] \\
= & \alpha p_{2}^{2}+W_{6} p_{2}+Y_{6},
\end{aligned}
$$

where $\alpha>0, W_{6}$ represents a coefficient, and $Y_{6}$ represents constant in this quadratic function.

Similar to Proposition 1, the profit functions $E\left(\Pi_{1}\right.$ । $\left.x_{1}, x_{0}\right)$ and $E\left(\Pi_{2} \mid x_{2}, x_{0}\right)$ are strictly concave in $p_{1}$ and $p_{2}$, respectively.

Proof of Proposition 4. According to (3), (4), and (41), we have

$$
\begin{aligned}
E & \left(\Pi_{3} \mid x_{0}\right) \\
= & E\left\{\left[d-(\alpha-\beta)\left(p_{1}+p_{2}\right)+2 \mu\right] p_{w} \mid x_{0}\right\} \\
= & \left\{d-(\alpha-\beta) E\left[\left(p_{1}+p_{2}\right) \mid x_{0}\right]+2 E\left(\mu \mid x_{0}\right)\right\} p_{w} \\
= & \left\{d-(\alpha-\beta)\left[\left(\theta_{1}+\delta_{1}\right)-\left(\theta_{2}+\delta_{2}\right) \Delta\right.\right. \\
& \left.\left.\quad+\left(\theta_{3}+\delta_{3}\right) E\left(p_{w} \mid x_{0}\right)\right]+2 \Delta\right\} p_{w} \\
= & (\beta-\alpha)\left(\theta_{3}+\delta_{3}\right) p_{w}^{2}+W_{7} p_{w}+Y_{7},
\end{aligned}
$$

where $\alpha>\beta, W_{7}$ represents a coefficient, and $Y_{7}$ represents constant in this quadratic function.

Similarly, according to the nature of the quadratic function, the profit function $E\left(\Pi_{3} \mid x_{0}\right)$ is strictly concave in $p_{w 1}$.
Proof of Proposition 5. According to (14), (15), and (41), we have

$$
\begin{aligned}
E & \left(\Pi_{3} \mid x_{0}\right) \\
= & E\left\{\left[d-(\alpha-\beta)\left(p_{1}+p_{2}\right)+2 \mu\right] p_{w} \mid x_{0}\right\} \\
= & \left\{d-(\alpha-\beta) E\left[\left(p_{1}+p_{2}\right) \mid x_{0}\right]+2 E\left(\mu \mid x_{0}\right)\right\} p_{w} \\
= & \left\{d-(\alpha-\beta)\left[\left(\theta_{1}+\delta_{1}\right)+\left(\theta_{2}+\delta_{2}\right) \Delta+\left(\theta_{3}+\delta_{3}\right)\right.\right. \\
& \left.\left.\times E\left(p_{w} \mid x_{0}\right)+\delta_{4} E\left(x_{0} \mid x_{0}\right)\right]+2 \Delta\right\} p_{w} \\
= & (\beta-\alpha)\left(\theta_{3}+\delta_{3}\right) p_{w}^{2}+W_{8} p_{w}+Y_{8},
\end{aligned}
$$

where $\alpha>\beta, W_{8}$ represents a coefficient, and $Y_{8}$ represents constant in this quadratic function.

Similarly, according to the nature of the quadratic function, the profit function $E\left(\Pi_{3} \mid x_{0}\right)$ is strictly concave in $p_{w 2}$.

Proof of Proposition 6. According to (30), (31), and (41), we have

$$
\begin{aligned}
E & \left(\Pi_{3} \mid x_{0}\right) \\
= & E\left\{\left[d-(\alpha-\beta)\left(p_{1}+p_{2}\right)+2 \mu\right] p_{w} \mid x_{0}\right\} \\
= & \left\{d-(\alpha-\beta) E\left[\left(p_{1}+p_{2}\right) \mid x_{0}\right]+2 E\left(\mu \mid x_{0}\right)\right\} p_{w} \\
= & \left\{d-(\alpha-\beta)\left[\left(\theta_{1}+\delta_{1}\right)+\left(\theta_{2}+\delta_{2}\right) \Delta\right.\right. \\
& +\left(\theta_{3}+\delta_{3}\right) E\left(p_{w} \mid x_{0}\right) \\
& \left.\left.\quad+\left(\theta_{4}+\delta_{4}\right) E\left(x_{0} \mid x_{0}\right)\right]+2 \Delta\right\} p_{w} \\
= & (\beta-\alpha)\left(\theta_{3}+\delta_{3}\right) p_{w}^{2}+W_{9} p_{w}+Y_{9}
\end{aligned}
$$

where $\alpha>\beta, W_{9}$ represents a coefficient, and $Y_{9}$ represents constant in this quadratic function.

Similarly, according to the nature of the quadratic function, the profit function $E\left(\Pi_{3} \mid x_{0}\right)$ is strictly concave in $p_{w 3}$.

\section{Conflict of Interests}

The authors declare that there is no conflict of interests regarding the publication of this paper.

\section{Acknowledgments}

The authors thank the editor, associate editor, and two anonymous reviewers for their constructive suggestions, which have improved this paper tremendously. This work is partially sponsored by the National Natural Science Foundation of China (71172174, 70772073) and the Ph.D. Programs Foundation of the Ministry of Education of China (20110075110003), the China Scholarship Council (201206630035), the Innovation Program of Shanghai Municipal Education Commission (12ZS58), and the Shanghai Pujiang Program (12PJC020). 


\section{References}

[1] K. Sadeddin, A. Serenko, and J. Hayes, "Online shopping bots for electronic commerce: the Comparison of functionality and performance," International Journal of Electronic Business, vol. 5, no. 6, pp. 576-589, 2007.

[2] D. Grewal, K. B. Monroe, and R. Krishnan, "The effects of pricecomparison advertising on buyers' perceptions of acquisition value, transaction value, and behavioral intentions," Journal of Marketing, vol. 62, no. 2, pp. 46-59, 1998.

[3] A. Serenko and J. Hayes, "Investigating the functionality and performance of online shoppingbots for electronic commerce: a follow-up study," International Journal of Electronic Business, vol. 8, no. 1, pp. 1-15, 2010.

[4] W. Huang and J. M. Swaminathan, "Introduction of a second channel: implications for pricing and profits," European Journal of Operational Research, vol. 194, no. 1, pp. 258-279, 2009.

[5] K. Cattani, W. Gilland, and J. M. Swaminathan, "Coordinating internet and traditional supply chains," in Handbook of Quantitative Supply Chain Analysis: Modeling in the E-Business Era, D. Simchi-Levi, D. Wu, and M. Shen, Eds., Elsevier, 2004.

[6] A. A. Tsay and N. Agrawal, "Manufacturer and reseller perspectives on channel conflict and coordination in multiplechannel distribution," in Handbook of Quantitative Supply Chain Analysis: Modeling in the E-Business Era, D. Simchi-Levi, D. Wu, and M. Shen, Eds., Elsevier, 2004.

[7] D.-Q. Yao and J. J. Liu, "Competitive pricing of mixed retail and e-tail distribution channels," Omega, vol. 33, no. 3, pp. 235-247, 2005.

[8] G. S. Cai, "Channel selection and coordination in dual-channel supply chains," Journal of Retailing, vol. 86, no. 1, pp. 22-36, 2010.

[9] J. Chen, H. Zhang, and Y. Sun, "Implementing coordination contracts in a manufacturer Stackelberg dual-channel supply chain," Omega, vol. 40, no. 5, pp. 571-583, 2012.

[10] K. S. Moorth, "Product and price competition in a duopoly," Marketing Science, vol. 7, no. 2, pp. 141-168, 1988.

[11] C. A. Ingene and M. E. Parry, "Channel coordination when retailers compete," Marketing Science, vol. 14, no. 4, pp. 360-377, 1996.

[12] W.-Y. K. Chiang, D. Chhajed, and J. D. Hess, "Direct marketing, indirect profits: a strategic analysis of dual-channel supplychain design," Management Science, vol. 49, no. 1, pp. 1-20, 2003.

[13] F.-F. Tang and X. Xing, "Will the growth of multi-channel retailing diminish the pricing efficiency of the web?" Journal of Retailing, vol. 77, no. 3, pp. 319-333, 2001.

[14] A. Dumrongsiri, M. Fan, A. Jain, and K. Moinzadeh, "A supply chain model with direct and retail channels," European Journal of Operational Research, vol. 187, no. 3, pp. 691-718, 2008.

[15] B. Dan, G. Xu, and C. Liu, "Pricing policies in a dual-channel supply chain with retail services," International Journal of Production Economics, vol. 139, no. 1, pp. 312-320, 2012.

[16] R. Desiraju and S. Moorthy, "Managing a distribution channel under asymmetric information with performance requirements," Management Science, vol. 43, no. 12, pp. 1628-1644, 1997.

[17] M. Cakanyildirim and Sethi, "Contracting underasymmetric information about production cost information," Working Paper, University of Texas at Dallas, Dallas, Tex, USA, 2007.

[18] S. K. Mukhopadhyay, X. Zhu, and X. Yue, "Optimal contract design for mixed channels under information asymmetry," Production and Operations Management, vol. 17, no. 6, pp. 641650, 2008.
[19] A. A. Tsay and N. Agrawal, "Channel dynamics under price and service competition," Manufacturing and Service Operations Management, vol. 2, no. 4, pp. 372-391, 2000.

[20] M. H. DeGroot, Optimal Statistical Decisions, Wiley Classics Library, Wiley-Interscience, Hoboken, NJ, USA, 2004.

[21] C. H. Chiu, T. M. Choi, and D. Li, "Price wall orpricewar: the pricing strategies for retailers," IEEE Transactions on Systems, Man, and Cybernetics A, vol. 39, no. 2, pp. 331-343, 2009.

[22] T. M. Choi, D. Li, and H. Yan, "Mean-variance analysis of a single supplier and retailersupply chain under a returns policy," European Journal of Operational Research, vol. 18, no. 4, pp. 356376, 2008.

[23] B. Shen, T. M. Choi, Y. Wang, and C. K. Y. Lo, "The coordination offashion supply chains with a risk averse supplier under the markdown money policy," IEEE Transactions on Systems, Man, and Cybernetics-Systems, vol. 43, no. 2, pp. 266-276, 2013.

[24] A. Ha and S. Tong, "Contracting and information sharing under supply chain competition," Management Science, vol. 52, no. 4, pp. 701-715, 2008.

[25] C. Chiu, T. Choi, H. Yeung, and Y. Zhao, "Sales rebate contracts in fashion supply chains," Mathematical Problems in Engineering, vol. 2012, Article ID 908408, 19 pages, 2012.

[26] M. Xu, Q. Wang, and L. Ouyang, "Coordinating contracts for two-stage fashion supply chain with risk-averse retailer and price-dependent demand," Mathematical Problems in Engineering, vol. 2013, Article ID 259164, 12 pages, 2013.

[27] J.-H. Zheng, B. Shen, P.-S. Chow, and C.-H. Chiu, "The impact of the strategic advertising on luxury fashion brands with social influences," Mathematical Problems in Engineering, vol. 2013, Article ID 534605, 16 pages, 2013. 


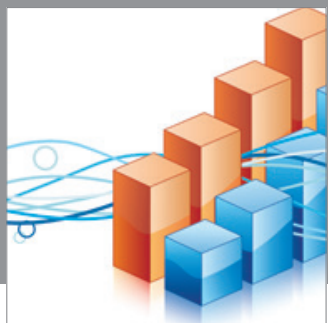

Advances in

Operations Research

mansans

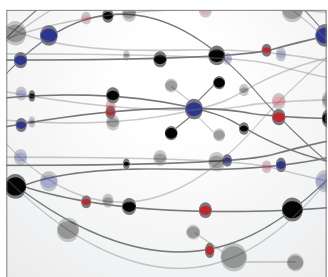

The Scientific World Journal
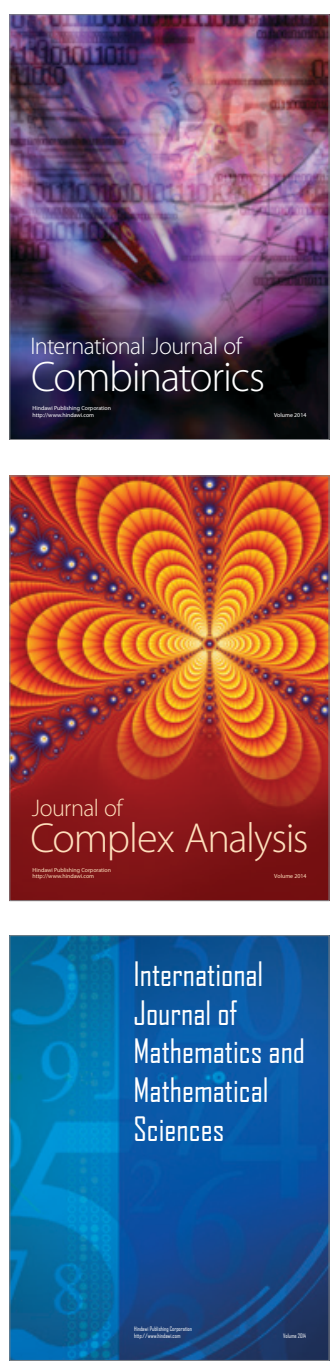
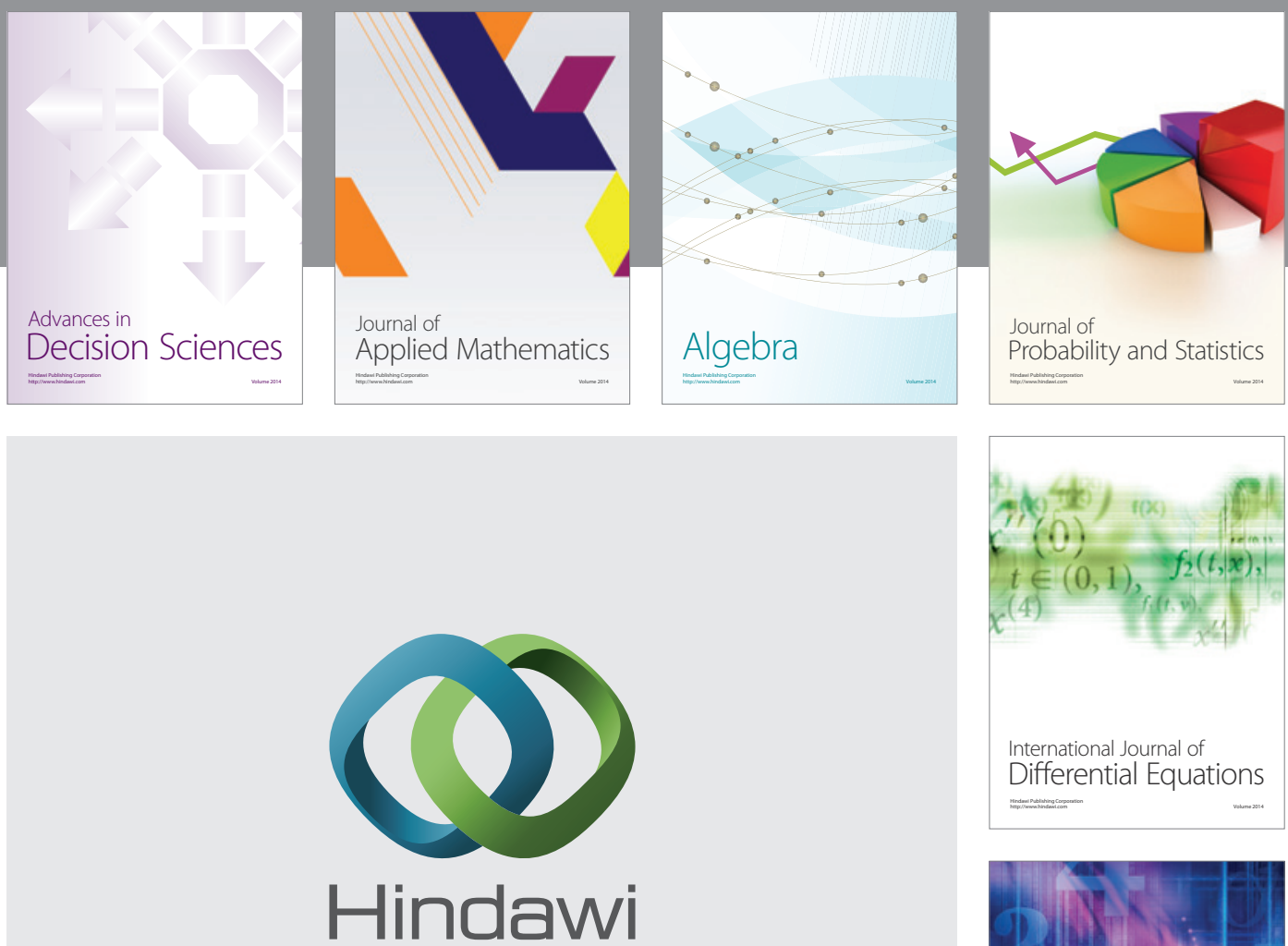

Submit your manuscripts at http://www.hindawi.com
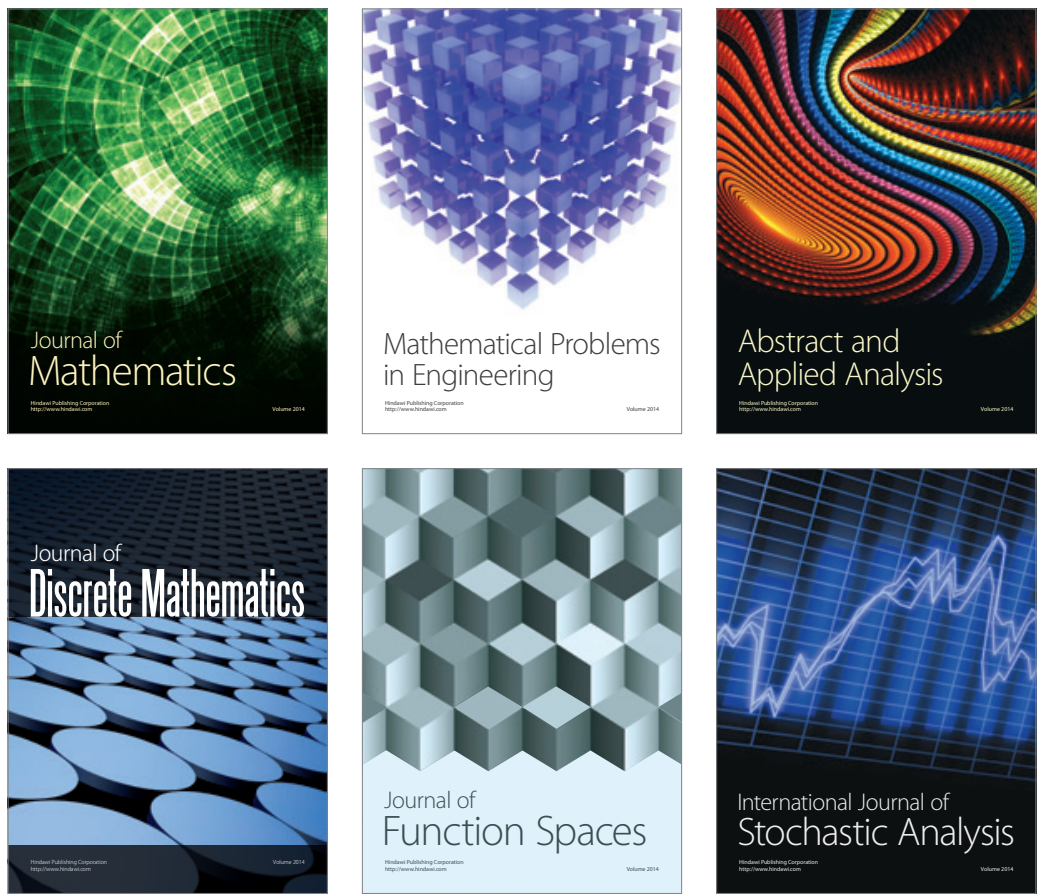

Journal of

Function Spaces

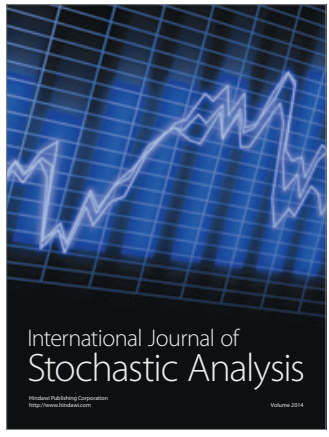

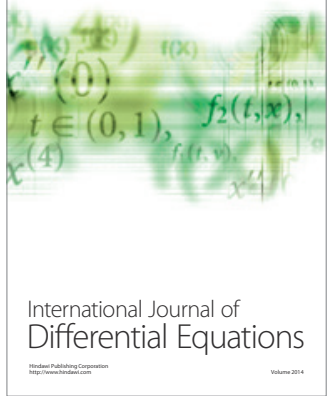
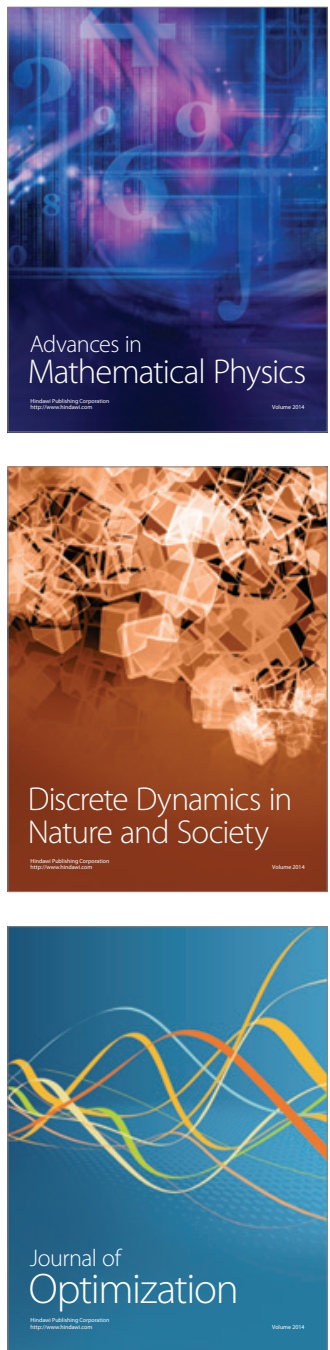